\title{
Raisonnements dans la résolution de problèmes d'électrocinétique par les étudiants de licence
}

Françoise Langlois et $\mathrm{J}$. Viard

\section{(2) OpenEdition}

1 Journals

Édition électronique

URL : http://journals.openedition.org/trema/2305

DOI : 10.4000/trema.2305

ISSN : 2107-0997

Éditeur

Faculté d'Éducation de l'université de Montpellier

\section{Édition imprimée}

Date de publication : 1 mai 1993

Pagination : 3-16

ISSN : 1167-315X

\section{Référence électronique}

Françoise Langlois et J. Viard, «Raisonnements dans la résolution de problèmes d'électrocinétique par les étudiants de licence », Tréma [En ligne], 3-4 | 1993, mis en ligne le 01 mai 1993, consulté le 04 mai 2019. URL : http://journals.openedition.org/trema/2305 ; DOI : 10.4000/trema.2305

Ce document a été généré automatiquement le 4 mai 2019.

Trema 


\title{
Raisonnements dans la résolution de problèmes d'électrocinétique par les étudiants de licence
}

\author{
Françoise Langlois et J. Viard
}

1 Le rapport entre la signification que l'on attribue à tel ou tel objet de pensée et l'utilisation qui est faite de cet objet dans la résolution d'un problème est un vaste sujet que nous n'aborderons pas de front mais qui sous-tend cependant l'étude présentée ici. La résolution d'un problème de physique doit-elle consister en un exercice de calcul formel, c'est à dire se limiter à la stricte application de règles de calcul à des symboles indépendamment de leur contenu sémantique? Ou bien au contraire la signification attribuée à ces symboles doit-elle être prise en compte dans le traitement auquel ils sont soumis lors de la résolution d'un problème ? Ces questions décisives n'ont pas fait l'objet d'un débat académique mais des étudiants s'y sont trouvés naturellement confrontés lors de la résolution de deux problèmes d'électrocinétique que nous leur avons soumis. La difficulté que beaucoup d'entre eux ont éprouvée dans la résolution du $1^{\mathrm{er}}$ problème les a conduits à s'interroger sur la pertinence des stratégies suivies d'où l'origine des questions précédentes. Les étudiants ont répondu à la fois par leurs discours et par les stratégies qu'ils mettaient en œuvre de fait dans la résolution d'un autre problème. Au traitement purement formel qui ignore les contenus sémantiques nous avons donné le nom de raisonnement algébrique. Au traitement qui s'appuie sur l'identité de l'objet manipulé, et sur ses propriétés caractéristiques nous donnons le nom de raisonnement qualitatif en nous référant à l'étymologie (qualis? = quel?, de quoi s'agit-il ? [Robert, rubrique qualité]).

Le travail que nous présentons ici a pour origine un enseignement de didactique sur les conceptions. Nous avons assuré successivement cet enseignement pendant trois années consécutives avec des effectifs respectifs de 15,13 et 29 étudiants. Les étudiants avaient pour la plupart une formation bac +2 option électronique ou électrotechnique (D.U.T ou B.T.S), et se destinaient à l'enseignement ; quelques uns étaient en licence de physique. Il 
s'agissait d'amener les étudiants à réfléchir sur les modes de raisonnements qu'ils utilisaient eux-mêmes et que leurs élèves pourraient aussi utiliser. Dans cette situation particulière les étudiants étaient conviés à résoudre une série d'exercices individuellement, anonymement et par écrit, les solutions fournies servant de base à une discussion collective. Compte tenu de la formation des étudiants nous avons choisi l'électrocinétique comme thème d'étude et nous avons utilisé quelques uns des tests mis au point par J.L Closset (1983) et S. Johsua et J.J. Dupin (1989). Dans le travail présenté ici nous n'en analysons que deux.

Le premier problème (ci-dessous) pose une question particulièrement intéressante. Ce problème met en œuvre le concept de tension ou de différence de potentiel (les deux termes étant considérés désormais comme équivalents) et sa relation avec l'intensité. L'étude des courbes de réponse à ce problème sur l'ensemble d'une scolarité (Johsua Dupin 1989) révèle une contradiction très nette entre un apprentissage manifeste relatif à l'égalité des tensions appliquées aux éléments des circuits (question $n^{\circ} 2$ ) et l'absence totale d'impact de cet apprentissage sur la valeur des intensités attribuées au courant traversant ces éléments (question $n^{\circ} 3$ ). L'absence de relation entre la tension aux bornes d'un élément et l'intensité traversant cet élément est résumée par les auteurs précédents par la formule très évocatrice : "La tension vit sa vie toute seule ». Ce constat suscite immédiatement la question: "Pourquoi la tension vit-elle sa vie toute seule?». Une réponse habituelle qui repose sur la conception du générateur comme un générateur de courant, bien qu'évidemment pertinente, nous a semblé insuffisante parce qu'elle demeure extérieure au concept de tension lui-même. Il nous a donc paru intéressant de rechercher d'autres éléments de réponse plus intrinsèquement liés au contenu du concept de tension mis en œuvre par les étudiants.

Problème $n^{\circ} 1$
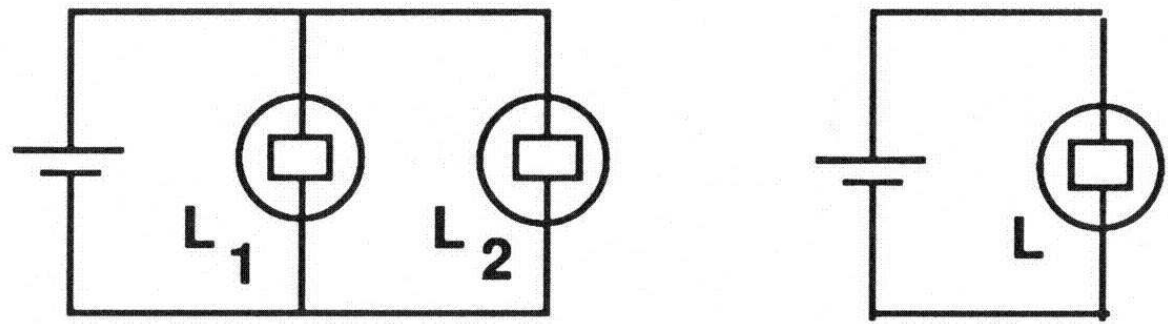

\author{
Que pensez-vous des phrases suivantes: \\ 1) Les différences de potentiel \\ mesurées aux bornes de \\ $\mathrm{L}_{1}$ et $\mathrm{L}_{2}$ sont égales. \\ 2)Les différences de potentiel mesurées \\ aux bornes de $\mathrm{L}_{1}$ \\ et $\mathrm{L}_{2}$ sont plus faibles que celles mesurées \\ aux bornes de $\mathrm{L}$. \\ 3)Les ampoules $\mathrm{L}_{1}$ et $\mathrm{L}_{2}$ brillent moins \\ fort que l'ampoule $\mathrm{L}$.
}

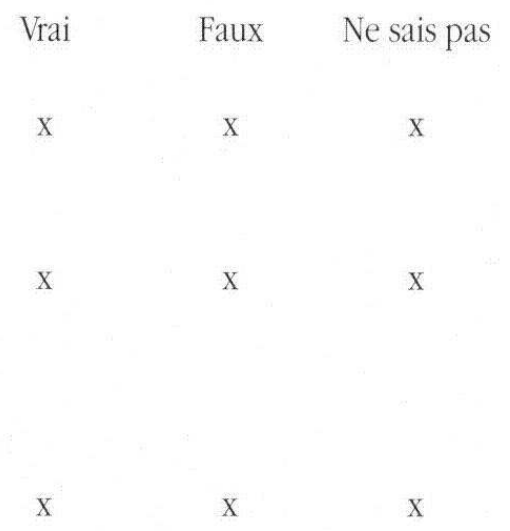


Le second problème (ci-dessous) a été élaboré par l'une d'entre nous (F. Langlois) à partir d'une question empruntée à J.L. Closset (1983) mais sensiblement modifiée. Alors que le premier problème est du type question à choix multiple, le second est un énoncé à réponses ouvertes qui ne contient aucune valeur numérique, ce qui peut favoriser le développement d'un raisonnement qualitatif. Les questions sont aussi posées en terme de variation qualitative seulement. La première fournit une observation et demande de l'interpréter, la seconde est une expérience de pensée et la question est posée en terme de prédiction. Ce problème présente également l'avantage de pouvoir être résolu par des stratégies très différentes. Une analyse des réponses possibles sera proposée plus loin.

Problème $n^{\circ} 2$

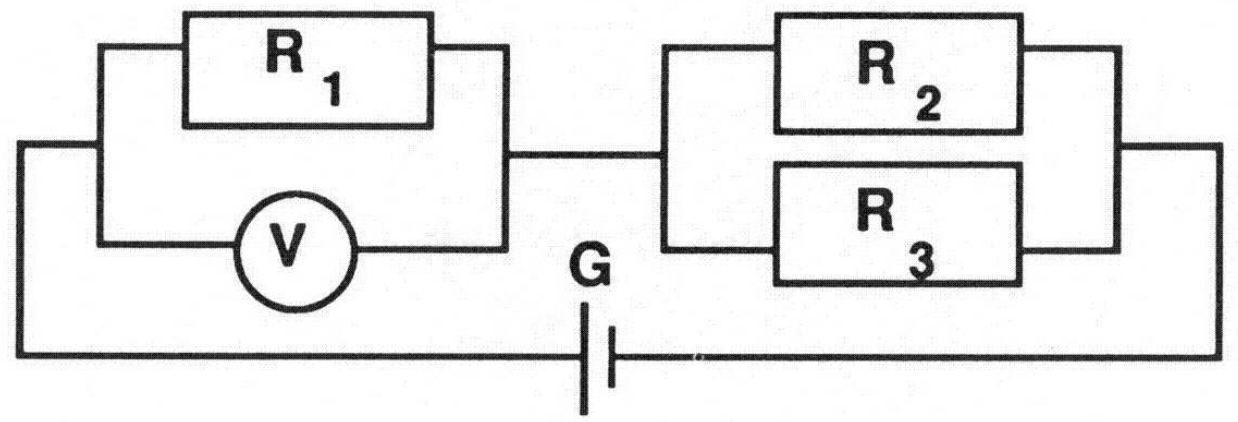

G est un générateur de résistance interne négligeable ; $R_{1}, R_{2}, R_{3}$ sont des résistances quelconques; $V$ un voltmètre de grande résistance interne.

a- On augmente la valeur de $\mathrm{R}_{1}$, on constate que la tension mesurée par le voltmètre augmente aussi.

Pourquoi?

b- On supprime $\mathrm{R}_{3}$. Comment varie la tension mesurée par le voltmètre ? Pourquoi ?

5 Les conditions de recueil des réponses et l'hétérogénéité des populations des différentes années enlèvent toute signification à un traitement quantitatif rigoureux de la répartition des étudiants entre différentes réponses. Nous nous sommes par la suite intéressés surtout à la typologie des réponses en mentionnant seulement dans certains cas et à titre indicatif le nombre de réponses obtenues pour chaque type.

6 Nous avons rencontré dans le traitement $\mathrm{du} \mathrm{Pb} \mathrm{n}^{\circ} 2$ des raisonnements à courant constant ou locaux mais en nombre minoritaire cependant. Les étudiants butant sur cet obstacle ne pouvant développer de stratégie de résolution un peu élaborée, nous avons laissé de côté ces réponses après les avoir recensées pour nous intéresser plutôt aux étudiants ayant surmonté cette difficulté. Nous proposons une caractérisation des stratégies efficaces, en mettant en évidence le mode de raisonnement utilisé (algébrique, qualitatif), les obstacles rencontrés. Notre hypothèse de travail concernant ce dernier point est que l'une des principales difficultés éprouvées par les étudiants dans la mise en œuvre d'un raisonnement qualitatif réside dans l'identification de l'objet à partir duquel ils raisonnent. Cette analyse pourra s'appliquer également aux difficultés rencontrées par les étudiants dans le $\mathrm{Pb} \mathrm{n}^{\circ} 1$.

7 L'ordre d'exposition sera désormais le suivant: après l'analyse a priori des réponses, nous examinerons les stratégies retenues par les étudiants pour la résolution $\mathrm{du} \mathrm{Pb} \mathrm{n}^{\circ} 2$ en nous efforçant de repérer l'incidence de la prise en compte du contenu sémantique des objets manipulés sur la pertinence de la solution retenue. Nous examinerons ensuite cette même relation entre le contenu du concept de tension pour les étudiants et le 
fonctionnement de ce concept comme outil de résolution $\mathrm{du} \mathrm{Pb} \mathrm{n}^{\circ} 1$. Un rapprochement sera fait avec l'élaboration historique du concept par Ohm dans les débuts de l'électrocinétique.

\section{Analyse a priori des réponses au deuxième problème}

\section{Réponses à la question - a}

- L'observation fournie par l'énoncé peut être interprétée de la façon suivante: la tension $\mathrm{V}$ mesurée par le voltmètre aux bornes de $\mathrm{R}_{1}$ dépend, selon la loi d'Ohm, du produit de la valeur de la résistance par la valeur de l'intensité. Si la tension aux bornes de $R_{1}$ s'accroît quand on augmente la valeur de la résistance $R_{1}$, c'est parce que la résistance augmente plus vite que l'intensité ne diminue. fonction de deux variables. Si l'on souhaite dépasser ce premier niveau de l'analyse factorielle, il est nécessaire d'envisager le circuit dans sa totalité. On peut dire alors que le résultat précédent est obtenu parce que la résistance $R_{1}$ contribue dans sa totalité à la détermination de $\mathrm{V}$ mais pour partie seulement à la détermination de la résistance totale du circuit qui fixe l'intensité. Les autres réponses supposent également une approche globale du circuit mais permettent de le ramener à des fonctions d'une seule variable.

a2 - Après avoir ramené le circuit à un circuit série et introduit la résistance équivalente aux résistances $R_{2}$ et $R_{3}$, l'application de la loi d'Ohm conduit à l'expression : $V=U_{G} R_{1}\left[\left(R_{1}\right.\right.$ $\left.+\mathrm{R}_{2} \mathrm{R}_{3} /\left(\mathrm{R}_{2}+\mathrm{R}_{3}\right)\right]^{-1}$

$\mathrm{U}_{\mathrm{G}}$ désignant la tension aux bornes du générateur $\mathrm{G}$, l'étude de la variation de $\mathrm{V}$ en fonction de $R_{1}$ fournit la réponse $: V$ augmente avec $R_{1}$. La seule variable explicite est la résistance.

$=0$ et $R_{1}$ infinie, la tension $\mathrm{V}$ prend alors respectivement les valeurs 0 et $\mathrm{U}_{\mathrm{G}}$; en considérant que $V\left(R_{1}\right)$ est continue et monotone, on déduit l'augmentation de $V$ avec $R_{1}$. La seule variable explicite est encore la résistance.

On considère le circuit comme un circuit série et on utilise deux propriétés caractéristiques d'un tel circuit alimenté par un générateur de tension: d'une part la somme des tensions sur l'ensemble du circuit est constante, d'autre part l'accroissement d'une résistance quelconque entraîne la réduction de l'intensité dans l'ensemble du circuit. Si $\mathrm{R}_{1}$ croît, l'intensité diminue, la chute de tension aux bornes de l'ensemble $\mathrm{R}_{2}, \mathrm{R}_{3}$ diminue, par suite $\mathrm{V}$ croît. La réponse ainsi exprimée peut aussi être formalisée au moyen de l'expression : $\mathrm{V}=\mathrm{U}_{\mathrm{G}}-\mathrm{R}_{\text {eq }} . \mathrm{I}(2)$ $R_{\text {eq }}=$ résistance équivalente à $R_{2}$ et $R_{3}$. La seule variable explicite est ici l'intensité. aussi », est considérée comme une propriété connue (et ne nécessitant pas de justification) du circuit précédent dénommé souvent « pont diviseur de tension » 


\section{Réponses à la question - $b$}

\section{Nous n'étudierons pas cependant systématiquement les réponses des étudiants question} par question mais plutôt leur mise en oeuvre dans une stratégie globale.

\section{Stratégies employées par les étudiants dans la résolution du $\mathrm{Pb} \mathrm{n}^{\circ} 2$}

\section{Un essai de classification}

Pour les étudiants de troisième année interrogés sur les raisons de l'incapacité de 45\% $\mathrm{d}^{\prime}$ entres eux à répondre correctement à la dernière question $\mathrm{du} \mathrm{Pb} \mathrm{n}^{\circ} 1$ la cause est entendue: "C'est parce que l'on a raisonné qualitativement, intuitivement au lieu d'utiliser la loi d'Ohm que l'on n'a pu répondre correctement à la question posée ».

Pourtant, si l'on examine les stratégies "gagnantes » suivies par ces mêmes étudiants et par leurs prédécesseurs dans la résolution $\mathrm{du} \mathrm{Pb} \mathrm{n}{ }^{\circ} 2$, les choses apparaissent moins simples. Très peu parmi les étudiants qui parviennent à la réponse exacte utilisent d'un bout à l'autre du problème une stratégie purement algébrique c'est à dire :

- Recensement des relations algébriques disponibles, ici loi d'Ohm appliquée à $\mathrm{R}_{1}$ et au circuit tout entier $\left(\mathrm{V}=\mathrm{R}_{\mathrm{l}} \mathrm{I} ; \mathrm{UG}=\left(\mathrm{R}_{1}+\mathrm{R}_{\mathrm{eq}}\right) \mathrm{I}\right)$ et valeur de la résistance équivalente à deux résistances en parallèle $\left(R_{e q}=R_{2} R_{3} /\left(R_{2}+R_{3}\right)\right)$.

- Dérivation à partir des ces trois relations d'une expression analytique de la tension $\mathrm{V}$ de la forme $\mathrm{V}=\mathrm{f}\left(\mathrm{R}_{1}, \mathrm{R}_{3}\right)$ en éliminant la variable intensité (cf Eq.(1)).

- Etude mathématique de la variation de $\mathrm{f}$ en fonction de $\mathrm{R}_{1} \mathrm{R}_{3}$ et conclusion.

Un seul étudiant a résolu ainsi les deux questions du problème (réponses $\mathrm{a} 2, \mathrm{~b} 1$ ). Le plus grand groupe des étudiants qui résolvent correctement le problème (une quinzaine) utilise une stratégie mixte. Cette stratégie comporte une étape de raisonnement algébrique : écriture de $V=f\left(R_{1}, R_{3}\right)$, étude de $V$ en fonction de $R_{1}$ par des arguments mathématiques du type : passage à la limite ou étude graphique ou étude de la variation 
du dénominateur d'une fraction (réponse a2). Cette première étape est suivie d'un raisonnement qualitatif (réponse b2), qui consiste à reconnaître que dans $V=f\left(R_{1}, R_{3}\right)$, l'expression $R_{2} R_{3} /\left(R_{2}+R_{3}\right)$ désigne une résistance équivalente et que cet objet possède une propriété physique spécifique rendant superflu le calcul de la variation de l'expression précédente avec $R_{3}$. Certains étudiants énoncent ainsi solennellement: "...la résistance équivalente à deux résistances en parallèle est toujours plus faible que la plus petite valeur des deux résistances. ", d'autres indiquent plus sommairement: « $R_{2}>R_{2} / / R_{3 »}$. Un autre groupe d'étudiants, cependant deux fois moins nombreux, en partant de la même relation $\left(\mathrm{R}_{2}>\mathrm{R}_{\mathrm{eq}}\right)$ est parvenu à la bonne prédiction pour la question $\mathrm{b}$ sans recours à une expression algébrique du type (1) mais en remarquant simplement que l'accroissement de la résistance en série avec $R_{1}$ entraîne une réduction de l'intensité dans le circuit (réponses b3) ou que la somme des tensions dans le circuit est constante (réponse b4). Deux étudiants ont répondu à la question a) en remarquant que la somme des tensions est constante et que l'accroissement de $\mathrm{R}_{1}$ entraine une réduction de la chute de tension aux bornes de $R_{2}, R_{3}$ (réponse a4). Enfin un étudiant seulement a résolu entièrement le problème par un raisonnement de type qualitatif, non formalisé (réponses a4 et b3).

Si maintenant nous examinons les procédures qui conduisent à un échec (en dehors de ceux qui raisonnent explicitement à courant constant ou localement), le plus grand nombre (une dizaine) est le fait d'étudiants appliquant la loi d'Ohm à la résistance $\mathrm{R}_{1}$ mais ne prenant en compte que le seul facteur «résistance» en ignorant l'incidence de l'intensité sur la valeur de la tension $\mathrm{V}$. Un certain nombre d'étudiants sont parvenus à une expression du type $V=f\left(R_{1}, R_{3}\right)$ mais ont été incapables ensuite de l'exploiter. Enfin un certain nombre d'étudiants qui ont tenté d'évaluer qualitativement l'effet de la suppression de la résistance $\mathrm{R}_{3} \mathrm{n}$ 'ont pas abouti à la bonne conclusion. Au vu de l'ensemble de ces données il est difficile de conclure avec les étudiants comme pour le $\mathrm{Pb}$ $\mathrm{n}^{\circ} 1$ que la raison d'échec dans l'élaboration d'une solution adéquate est liée au choix d'un mode de raisonnement qualitatif puisque certains qui avaient choisi ce mode de raisonnement sont parvenus à la solution et d'autres pas (de même ceux qui ont choisi un autre mode de raisonnement ont réussi pour partie seulement). S'il n'est pas possible de trancher en faveur de l'une ou l'autre stratégie on peut remarquer cependant que l'une et l'autre présentent des avantages et des inconvénients, des difficultés de mise en œuvre spécifiques.

\section{Avantages et inconvénients des deux stratégies}

La stratégie qui consiste à exprimer analytiquement la grandeur $\mathrm{V}$ en fonction des variables explicites de l'énoncé $\left(\mathrm{R}_{1}, \mathrm{R}_{3}\right)$ est sûre et facile à mettre en œuvre par les étudiants qui raisonnent sur l'ensemble du circuit. Elle permet d'éliminer sans subtilité l'une des variables dont dépend la grandeur $\mathrm{V}$ et de faire disparaître ainsi une difficulté de la question $\mathrm{a}: \mathrm{V}=\mathrm{R}_{1} \mathrm{I}$ dépend de deux facteurs dont les effets peuvent se compenser l'un l'autre (ce qui a conduit des étudiants à affirmer que, contrairement à l'énoncé, $V$ restait constante). Une fois l'expression $V=f\left(R_{1}, R_{3}\right)$ obtenue, l'obstacle éventuel ne peut venir que des mathématiques, ici l'étude de la variation d'une fonction numérique. En aucun cas la signification que l'on attribue aux grandeurs désignées par cette fonction n'a à intervenir dans le traitement mathématique dont cette fonction est l'objet, ce qui écarte les difficultés d'interprétation. En contrepartie cette procédure présente au moins deux inconvénients : tout d'abord elle est lourde, couteuse en étapes de raisonnement ; ainsi 
l'étude de la variation de $V$ avec $R_{1}$ et $R_{3}$ est résolue en quatre étapes de calcul par l'étudiant qui a utilisé cette méthode. D'autre part elle est rigide : dans le cas présent elle impose l'élimination de la variable intensité alors que l'emploi de cette dernière permet de résoudre beaucoup plus judicieusement le problème. Si l'on admet les remarques précédentes il n'apparaît pas surprenant que le mode de raisonnement algébrique ait été utilisé par la grande majorité des étudiants (16 contre 3) qui ont résolu la question a. Une méthode sûre, même coûteuse, sera plus facilement choisie face à une situation délicate.

La deuxième stratégie utilisée pour résoudre la question a est beaucoup plus légère mais elle est également plus subtile dans la mesure où elle nécessite un détour. $\mathrm{V}$ n'est pas obtenue directement à partir de $\mathrm{R}_{1}$ mais par l'intermédiaire de la tension aux bornes de $\mathrm{R}$ ${ }_{2}, \mathrm{R}_{3}$ soustraite à la tension fournie par le générateur qui est constante. Cette quantité soustraite comme l'intensité est une fonction décroissante de $\mathrm{R}_{1}$. Ce détour rend ainsi la dépendance de $\mathrm{V}$ à l'égard de $\mathrm{R}_{1}$ immédiatement apparente sans nécessiter aucun calcul. En contrepartie cette stratégie nécessite de prendre en compte la signification de la grandeur manipulée et les propriétés qui en découlent; dans l'exemple actuel $\mathrm{V}$ désigne une tension partielle dans un circuit série et la somme de ces tensions est conservée si le générateur est un générateur de tension. Dans la démarche algébrique les dépendances entre les grandeurs sont prises en compte implicitement dans les expressions formelles dont on part ou que l'on obtient à partir des premières en appliquant mécaniquement des règles de calcul. Ici au contraire, lorsqu'il y a une formalisation du raisonnement (cf. expression (2)), cette formalisation intervient à la fin pour synthétiser, exprimer sous une forme ramassée un ensemble de dépendances conceptuelles qui ont été repérées au préalable. Le raisonnement qualitatif fait donc nécessairement une place à l'interprétation puisqu'il comporte une étape déterminante d'identification des objets rencontrés. La pierre de touche d'une identification réussie est l'attribution à l'objet de ses qualités spécifiques, des bonnes propriétés. L'identification d'une grandeur, d'un objet physique n'est pas sans difficulté comme le montre le traitement de la deuxième question.

\section{Un exemple d'obstacle rencontré dans un raisonnement qualitatif}

Une trentaine d'étudiants, pour évaluer l'incidence de la suppression d'une résistance en parallèle sur la résistance totale du circuit, ont choisi de ne pas faire le calcul complet et d'argumenter en puisant dans leurs connaissances. Ces étudiants se répartissent en trois groupes :

- Un premier groupe majoritaire (23 étudiants) considère implicitement ou explicitement que $\mathrm{R}_{2}>\mathrm{R}_{\mathrm{eq}}$ et l'un d'entre eux énonce : « La somme de deux résistances en parallèle a toujours une valeur plus petite qu'une résistance.».

- Un groupe de 4 étudiants considère que : « Dans certains cas, $\mathrm{R}_{\text {eq }}=\mathrm{R}_{2} \mathrm{R}_{3} /\left(\mathrm{R}_{2}+\mathrm{R} 3\right)<\mathrm{R} 2$ et, dans d'autres cas, $R_{\text {eq }}=R_{2} R_{3} /\left(R_{2}+R_{3}\right)>R_{2}$, cela dépend de la valeur de $R_{3}$ par rapport à $R_{2}$ ou à $R_{\text {eq }}$. "

- Enfin 3 étudiants considèrent que dans tous les cas $\mathrm{R}_{\mathrm{eq}}>\mathrm{R}_{2}$; ils s'expriment ainsi : « [...] $\mathrm{RT}=\mathrm{R}$ ${ }_{1}+R_{\text {eq }}[\ldots]$ on supprime $R_{3}$, RT décroit. »; « $R_{3}$ enlevée, $R T$ diminue »; « La tension augmente car supprimer $R_{3}$ revient à diminuer la résistance $R_{2} / / R_{3}$ et donc la résistance totale $d u$ circuit ».

Il apparaît clairement que la notion de résistances en parallèle n'est pas la même pour tous les étudiants. En supposant qu'une grandeur peut être distinguée d'une autre par ses propriétés d'additivité, nous nous trouvons dans le cas $n^{\circ} 1$ et dans le cas $n^{\circ} 3$ en présence 
de deux grandeurs complètement différentes. Dans le dernier cas les résistances en parallèle obéissent aux règles communes de l'addition: «supprimer une résistance en parallèle revient à diminuer la résistance totale du circuit». Pour l'étudiant cité du premier groupe au contraire les résistances en parallèle obéissent à des règles d'addition tout à fait particulières et paradoxales puisque la somme est plus petite qu'un seul terme. Les étudiants du deuxième groupe savent que les règles habituelles de l'addition ordinaire ne s'appliquent pas mais ne connaissent pas les nouvelles règles. Il semble alors que la conceptualisation pour l'étudiant du premier groupe a consisté à passer du stade du groupe trois -qui considère les résistances en parallèle comme un objet banal auquel on applique les règles du sens commun- à un autre stade qui reconnaît la particularité de cet objet singulier. Le paradoxe de l'addition des résistances en parallèle aurait pu être levé facilement en introduisant les notions de conduction et de conductance mais ces notions ne semblaient pas faire partie de l'enseignement qu'avaient reçu les étudiants. L'interprétation d'un échec dans un raisonnement qualitatif en terme d'erreur sur la nature de l'objet physique rencontré dans la résolution d'un problème peut maintenant être étendue au cas $\mathrm{du} \mathrm{Pb} \mathrm{n}^{\circ} 1$ et au concept de tension.

\section{Un malentendu sur le concept de tension électrique}

30 L'idée proposée est la suivante : si un nombre important d'étudiants a conclu à l'égalité des tensions aux bornes de la lampe $\mathrm{L}$ et des lampes $\mathrm{L}_{1}$ et $\mathrm{L}_{2}$, tout en affirmant que les dernières brillaient moins que la lampe $\mathrm{L}$ ce n'est pas parce que les étudiants ont raisonné qualitativement ou parce qu'ils n'ont pas une bonne connaissance de la loi d'Ohm mais parce qu'ils n'ont pas acquis le bon concept de tension. Ces étudiants ont acquis une connaissance relative à l'égalité des grandeurs, mais n'ont pas acquis une connaissance relative à la signification, à l'identité de la grandeur mesurée. Cette supposition nous a conduit à interroger par écrit les étudiants sur le contenu qu'ils attribuaient à ce concept en posant la question suivante : «Qu'est-ce-que la tension ou la différence de potentiel ?»

31 La formulation de la question avait pour but d'écarter des réponses tautologiques de la forme : la tension c'est la différence de potentiel : cependant sur 23 réponses recueillies nous avons malgré cette précaution obtenues 4 réponses de ce type. Une autre réponse se limite à indiquer que l'unité de tension est le volt. Deux réponses indiquent que la tension est une différence entre deux valeurs sans plus de précision. 16 réponses plus explicites se répartissent comme suit :

- Trois réponses mettent directement la tension en relation avec un « courant », « un déplacement », « une circulation de charge électrique » par exemple : «[...] la circulation des électrons entre deux points implique que les deux points sont à des potentiels différents, la tension est dans le sens de circulation des électrons. »

- Quatre réponses font référence à une différence de niveau dans un circuit, l'une précise : " une différence de niveau électrique entre deux points d'un circuit ».

- Une réponse identifie la tension à la «force électrique entre deux points »

- Huit réponses donnent une définition purement statique en terme de différence de charge électrique présente en deux endroits distincts sans aucune référence au déplacement éventuel de ces charges, à leur mobilité.

32 En résumé : trois réponses donnent au concept un contenu directement applicable à un problème d'électrocinétique. Les réponses en termes de niveau peuvent être associées à l'idée d'un nivellement et d'un écoulement de charge. Mais huit réponses sur les seize 
explicites donnent au concept une connotation purement électrostatique. La fonction désignée est le stockage de l'électricité et le modèle sous-jacent le condensateur. Deux tensions identiques signifient une égalité de la charge accumulée. La problématique évoquée et la fonction attribuée au concept de tension excluent a priori son emploi dans un problème d'électrocinétique. Il n'apparaît donc pas déraisonnable de penser que si dans le $\mathrm{Pb} \mathrm{n}^{\circ} 1$ l'évocation de l'égalité de deux tensions n'a pas entrainé l'utilisation de la loi d'Ohm c'est parce que cette loi en dehors de son aspect quantitatif implique une mise en relation explicite du concept de tension et du concept d'écoulement de charge électrique : alors que cette relation était exclue de la conception qu'avait un certain nombre d'étudiants de la tension électrique. L'enquête précédente en raison de son caractère très limité ne permet pas à elle seule de garantir le bien fondé de l'interprétation proposée du comportement des étudiants et une autre expérimentation qui n'a pu être réalisée jusqu'à maintenant serait nécessaire. Toutefois l'existence de deux concepts distincts de la tension électrique est attestée cette fois sans ambiguïté au niveau de l'élaboration historique du concept et ce fait à lui seul mérite de retenir l'attention. En effet il peut d'une part faciliter l'acceptation de l'existence d'une telle dualité chez les étudiants et d'autre part rendre compréhensible la subsistance de cette séparation dans l'enseignement.

\section{La relation entre tension et conduction avant Ohm et les premiers fondements de l'électrodynamique}

33 Avant Ohm : le seul cadre conceptuel dans lequel est définie, utilisée la tension électrique est celui de l'électrostatique. La tension est reliée à une densité de charges accumulées dans un espace donné : «[...] cette force (la tension) est proportionnelle à la densité du fluide ou au nombre de molécules renfermées dans un espace donné » (Haüy, 1803).

La tension caractérise principalement l'état d'un condensateur chargé (bouteille de Leyde) et l'existence de charges de signes opposées à ses bornes. Elle porte alors le nom «d'intensité statique» (Pourprix, Locqueneux, 1988). Elle est mesurée au moyen d'un électroscope à feuilles d'or. Les physiciens de l'époque n'ignorent pas les phénomènes de conduction et le principal intérêt de l'accumulation de l'électricité dans un condensateur réside dans la décharge qui va suivre. Mais le seul état à être caractérisé physiquement au moyen de grandeurs physiques (tension, quantité de charges) associées à un paramètre mesurable (écartement des feuilles de l'électroscope) est l'état stable initial du condensateur chargé. La décharge, phénomène transitoire, trop bref, n'est pas vraiment caractérisée, mais observée. Enfin la seule relation établie entre la tension et la conduction est une relation d'opposition, d'exclusion mutuelle (Benséghir, 1989) : quand on relie les bornes d'un condensateur par un conducteur la tension disparaît. La découverte de la pile de Volta et de l'effet magnétique de l'électricité par Oersted ouvrent de nouvelles possibilités expérimentales et théoriques: accroissement de la durée de conduction et possibilité de caractériser un écoulement de charges par l'intensité de son effet magnétique (galvanomètre). Mais la structure conceptuelle à travers laquelle les nouveaux phénomènes sont analysés, interprétés, reste celle de l'électrostatique. La pile est assimilée à un condensateur qui se recharge constamment : «[...] aussi une pile n'estelle autre chose qu'une bouteille de Leyde qui aurait la propriété de se recharger ellemême aussitôt qu'elle serait déchargée. » (Thénard, 1813). 

considérée comme un condensateur : «si on joint les deux extrémités d'une pile par un bon conducteur de l'électricité, les phénomènes de tension disparaissent entièrement » (Colladon 1826) l'électrodynamique et ses effets : «[...] dans le cas de l'électricité en mouvement [...] il n'y a pas plus de tension électrique mais quand on interrompt le circuit [...] les tensions se rétablissent elles-mêmes, tout ceci prouve tout à fait bien que les tensions ne causent ni la décomposition de l'eau ni le changement de direction de l'aiguille magnétique.» (Ampère 1820).

Ohm opère alors une révolution conceptuelle (Shagrin 1963) en mettant l'accent non plus sur l'opposition mais sur la relation nécessaire entre l'existence d'une différence de potentiel (différence de force électroscopique) et un déplacement d'électricité. La conduction n'est pas autre chose que le rétablissement d'un équilibre préalablement rompu entre les forces électroscopiques d'éléments électriques voisins. La conduction n'existe que tant que le déséquilibre subsiste. Il étudie localement le phénomène de conduction aussi bien expérimentalement que théoriquement et conclut ainsi : "Quand deux éléments électriques $E$ et $E^{\prime}$, d'égale grandeur et de même forme, semblablement placés l'un par rapport à l'autre, mais doués de force électroscopiques d'inégales intensités, sont situés à distance convenable l'un de l'autre, ils manifestent une tendance mutuelle vers l'état d'équilibre; ils vont continuellement en se rapprochant de la moyenne de leurs états électriques, jusqu'à ce qu'ils l'aient atteinte. C'est-à-dire que l'état électrique de ces éléments se modifie aussi longtemps qu'il existe une différence entre leurs forces électroscopiques, mais cet état devient invariable quand les deux éléments sont arrivés à la même force électroscopique. Par conséquent la variation de l'état électrique et la différence des forces électroscopiques sont deux quantités liées entre elles de telle manière qu'elles disparaissent en même temps. » (Ohm 1827).

Le renversement de point de vue opéré par Ohm apparaît explicitement dans la dernière phrase : «différence de force électroscopique » et "variation d'état électrique » [...] "disparaissent en même temps ». Même si la terminologie employée pour désigner la grandeur (force électroscopique) et l'appareil utilisé pour la mesurer (l'électroscope) évoquent le potentiel électrostatique, le concept mis en oeuvre par Ohm est cependant entièrement nouveau en ce sens qu'il est défini non par référence à une quantité de charges immobiles mais par référence à une quantité d'électricité transportée en un temps donné, à un flux d'électricité.

'enoncé précédent dans sa formulation même en rappelle un autre réalisé peu avant par Carnot et relatif au transport de la chaleur: "La production du mouvement dans les machines à vapeur est toujours accompagnée d'une circonstance sur laquelle nous devons fixer l'attention. Cette circonstance est le rétablissement de l'équilibre dans le calorique, c'est à dire son passage d'un corps où la température est plus ou moins élevée à un autre où elle est plus basse. » (Carnot 1824)

40 La référence commune à la «tendance à l'état d'équilibre » et implicitement au second principe de la thermodynamique fonde théoriquement la possibilité d'une analogie entre le transport de la chaleur et celui de l'électricité. Cette analogie sera traduite ensuite dans la similitude des équations locales de conduction : «J'ai admis que la grandeur du passage d'électricité entre deux éléments contigus est proportionnelle à la différence de force 
électroscopique que possèdent les deux éléments, de même manière que dans la théorie de la chaleur on considère le passage de la chaleur entre deux éléments comme proportionnel à la différence de leur température. » $(\mathrm{Ohm}, 1827)$.

41 La grandeur qui exprime le rapport entre le flux d'électricité et le gradient de force électroscopique est la conductibilité du matériau : $\mathrm{I} / \mathrm{S}=\mathrm{k}(\mathrm{du} / \mathrm{dx})$ (Pourprix, Locqueneux, 1988) $\mathrm{k}$ désigne la conductibilité électrique, $\mathrm{u}$ la force électroscopique à l'abscisse $\mathrm{x}, \mathrm{S}$ la section du conducteur.

Le changement conceptuel opéré par Ohm est à l'origine de l'opposition que son travail a rencontré chez ses contemporains comme le souligne Shagrin (1963) : «Ohm paraît confondre la distinction bien établie entre tension électrique et courant électrique. » [...] "Sa tentative de réunir en une seule formule deux phénomènes distincts rencontre une formidable opposition. ».

Son contradicteur le plus acharné G.F. Pohl (1828) lui reproche de remplacer l'électroscope par un ampèremètre : «[...] l'aiguille magnétique en vint soudainement à représenter la fonction de l'électroscope [...] »

Aujourd'hui le fait qu'un ampèremètre soit un voltmètre ne choque plus personne mais il n'est pas certain que les implications conceptuelles fortes sous-jacentes soient bien perçues pour autant. Si l'on compare l'apport original d'Ohm et la perception que l'on peut en avoir à partir de la présentation de la «loi » qui porte son nom dans l'enseignement de l'électrocinétique, trois différences essentielles sautent aux yeux :

- l'analyse locale, élémentaire, du phénomène de conduction est entièrement gommée au profit d'une approche semi-intégrale : la conduction envisagée non pas en un point mais sur toute une portion du circuit est symbolisée plutôt que modélisée par la donnée de la résistance entre deux points.

- l'introduction de cette boite noire qu'est la résistance interdit la conceptualisation de la relation entre la différence de potentiel et le flux de charge à travers la caractérisation du pouvoir de conduction du matériau : « La loi d'Ohm sous sa forme bien connue E = RI, peut laisser croire que la résistance $R$ du conducteur a joué un rôle essentiel dans l'élaboration de la théorie. Il n'en est rien : c'est dans la conductibilité k qu'est inscrite la conceptualisation première du phénomène de propagation » (Pourprix, Locqueneux, 1988).

- le cadre théorique plus large, principe d'un rétablissement des équilibres à l'origine des flux, qui fonde la relation entre différence de potentiel et courant, est totalement absent.

En d'autres termes on retient d'Ohm le constat d'une relation de proportionnalité entre deux grandeurs définies empiriquement chacune séparément. Mais tous les fondements conceptuels, issus d'une étude physique de la conduction, qui pouvaient justifier l'existence, et la construction d'une telle relation entre ces grandeurs et lui donner un sens, ont été supprimés.

\section{Conclusion}

L'utilisation de questions ouvertes s'est avérée un outil très efficace pour révéler la richesse imaginative des étudiants et la diversité des stratégies employées pour résoudre un problème d'électrocinétique qui pouvait sembler banal a priori. En analysant ces stratégies il apparaît que celles les plus fréquemment employées avec succès utilisent simultanément des modes de raisonnement algébrique et qualitatif. Contrairement à ce que pensent les étudiants, les raisonnements qualitatifs ne sont pas nécessairement les 
moins performants. Lorsqu'ils conduisent à des échecs, les erreurs rencontrées ne sont pas dues au mode de raisonnement mais au fait que celui-ci est mal fondé et repose sur un ensemble de propriétés qui ne correspond pas à l'objet visé. Le raisonnement qualitatif nécessite pour sa mise en œuvre des bases conceptuelles solides. Un certain nombre d'erreurs peuvent être attribuées en effet à des confusions ou à des troncatures conceptuelles. En particulier la non référence à la tension dans des problèmes d'électrocinétique peut être imputée à une présentation et une perception exclusivement électrostatiques de la tension. Cette difficulté n'est pas nouvelle puisque Ohm y avait été déjà confronté. Il paraît par suite dangereux d'introduire cette grandeur dans l'enseignement indépendamment des phénomènes de transport et en se référant exclusivement à l'accumulation de charges électriques immobiles.

\section{BIBLIOGRAPHIE}

\section{Références}

AMPERE A.M., Ann. Chem. Phys., 15, cité par Shagrin in Ref ci-après, 1820.

BENSEGHIR A. : « Formation des concepts d'électrocinétique », Bulletin de l'Union des Physiciens, 713, pp. 451-465, 1989.

CARNOT S., (1824), Réflexions sur la puissance motrice du feu, Paris Gauthiers-Villars, 1878.

CLOSSET J.L., Le raisonnement séquentiel en électrocinétique. Thèse de $3^{\circ}$ cycle. Université Paris $7,1983$.

HAÜY R., Traité élémentaire de Physique (1803), cité par A. Benséghir in Ref. supra.

COLLADON J.D., (1826), Ann. Chem. Phys., 33, 62-65 cité par Shagrin in Ref ci-après

JOHSUA S., DUPIN J.J., Représentation et modélisation : le débat scientifique dans la classe et l'apprentissage de la physique. Peter Lang éditeur. Berne, 1989.

OHM G.S., (1827), Die galvanische Kette, mathematisch bearbeitet, Berlin ; traduit par J-M. GAUGAIN, Théorie mathématique des courants électriques, Paris, (1860). cité par Pourprix et Locqueneux in Ref. plus bas.

POHL G.F., (1828), Jahrb. Wissenschaftliche Kritik, 11, 85-104 cité par Shagrin cf Ref plus bas.

POURPRIX B., LOCQUENEUX R., G.S. Ohm et la théorie mathématique du circuit galvanique (1827), Fundamente Scientiae, Vol.9, №4, pp. 409-427, 1988.

SHAGRIN M.L., Resistance to Ohm's Law, Am. J. Phys., 31, 536-47, 1963.

THÉNARD L.J., Traité élémentaire de chimie (1813), cité par A. Benséghir in Ref. supra. 


\section{RÉSUMÉS}

La résolution d'un problème de physique doit-elle consister en un exercice de calcul formel ou bien prendre en compte la signification attribuée aux symboles utilisés? Nous tentons de répondre à cette question à partir d'exercices d'électrocinétique (niveau bac+2) mettant en jeu les concepts de résistance équivalente et de tension.

Is the problem solving in physics only an exercise of calculus with mathematic symbols (algebric reasoning) or is it necessary to know the significant of the symbols to solve the problem (qualitative reasoning)? We try to answer this question with students at university level. We ask them exercises using the concepts of voltage and shunt.

\section{INDEX}

Mots-clés : électrocinétique, raisonnement algébrique, raisonnement qualitatif, résolution de problème

\section{AUTEURS}

\section{FRANÇOISE LANGLOIS}

Université Claude Bernard de Villeurbanne, Lirdis

\section{J. VIARD}

Université Claude Bernard de Villeurbanne, Lirdis 